\title{
The Influence of Histologic Grade on Outcomes of Elderly Women With Early Stage Breast Cancer Treated With Breast Conserving Surgery With or Without Radiotherapy
}

\author{
Chase E. Escott ${ }^{1}$, David Zaenger ${ }^{2}$, Jeffrey M. Switchencko ${ }^{3}$, Jolinta Y. Lin ${ }^{1}$, Mustafa \\ Abugideiri ${ }^{1}$, Cletus A. Arciero ${ }^{4}$, Neil T. Pfister ${ }^{1}$, Karen M. Xu ${ }^{1}$, Jane L. Meisel ${ }^{5}$, Preeti \\ Subhedar $^{4}$, Mylin Torres ${ }^{1}$, Walter J. Curran ${ }^{1}$, Pretesh R. Patel ${ }^{1}$ \\ ${ }^{1}$ Department of Radiation Oncology, Winship Cancer Institute, Emory University, Atlanta, GA \\ ${ }^{2}$ Department of Radiation Oncology, Carolina Regional Cancer Center, Myrtle Beach, SC \\ ${ }^{3}$ Department of Biostatistics and Bioinformatics, Winship Cancer Institute, Emory University, \\ Atlanta, GA \\ ${ }^{4}$ Department of Surgical Oncology, Winship Cancer Institute, Emory University, Atlanta, GA \\ ${ }^{5}$ Department of Medical Oncology, Winship Cancer Institute, Emory University, Atlanta, GA
}

\section{Abstract}

Grade 3 disease was not well-represented in clinical trials investigating the omission of radiotherapy following breast conserving surgery in elderly women with early stage breast cancer. This Surveillance, Epidemiology, and End Results analysis of 12,036 women aged 70 to 79 years with T1N0, estrogen receptor-positive, invasive ductal carcinoma found that women with grade 3 disease had both an overall survival and breast cancer-specific mortality benefit with radiotherapy.

Background: Two large randomized trials, CALGB 9343 and PRIME II, support omission of radiotherapy after breast conserving surgery (BCS) in elderly women with favorable-risk early stage breast cancer intending to take endocrine therapy. However, patients with grade 3 histology were underrepresented on these trials. We hypothesized that high-grade disease may be unsuitable for treatment de-escalation and report the oncologic outcomes for elderly women with favorable early stage breast cancer treated with BCS with or without radiotherapy.

Materials and Methods: The Surveillance, Epidemiology, and End Results database was queried for women between 70 and 79 years of age with invasive ductal carcinoma diagnosed between 1998 and 2007. This cohort was narrowed to women with T1mic-T1c, N0, estrogen receptor-positive, invasive ductal carcinoma treated with BCS with or without external beam radiation (EBRT). The primary endpoints were 5- and 10-year cause-specific survival (CSS). Univariate and multivariate analyses were performed. Propensity-score matching of T-stage, year of diagnosis, and age was utilized to reduce selection bias while comparing treatment arms within the grade 3 subgroup.

Address for correspondence: Chase E. Escott, MD, Department of Radiation Oncology, Winship Cancer Institute, Emory University, Atlanta, GA 30322, contact: ceescot@emory.edu. 
Results: A total of 12,036 women met inclusion criteria, and the median follow-up was 9.4 years. EBRT was omitted in $22 \%$ of patients, including $21 \%$ with grade 3 disease. Patients in the EBRT cohort were slightly younger (median, 74 vs. 75 years; $P<.01$ ) and had fewer T1a tumors $(11 \%$ vs. $13 \% ; P=.02)$. Histologic grades 1,2 , and 3 comprised $36 \%, 50 \%$, and $14 \%$ of the cohort, respectively, and there were no differences in EBRT utilization by grade. Utilization of EBRT decreased following the publication of the CALGB trial in 2004 decreasing from $82 \%$ to $85 \%$ in 1998 to 2000 to $73 \%$ to $75 \%$ in 2005 to $2007(P<.01)$. Unadjusted outcomes showed that in grade 1 disease, there were no differences in CSS with or without EBRT at 5 (99\%) and 10 years (95\%-96\%). EBRT was associated with an improvement in CSS in grade 2 histology at 5 years $(97 \%$ vs. $98 \%)$ and 10 years $(92 \%$ vs. $95 \%)(P=.004)$. The benefit was more pronounced in grade 3 disease with CSS increasing from $93 \%$ to $96 \%$ at 5 years and from $87 \%$ to $92 \%$ at 10 years $(P=.02)$ with EBRT. In the grade 3 subgroup, propensity-score matching confirmed EBRT was associated with superior CSS compared with surgery alone (hazard ratio, $0.58 ; 95 \%$ confidence interval, $0.34-0.98 ; P=.043)$.

Conclusion: In this database analysis, omission of radiotherapy after BCS in elderly women with favorable-risk, early stage, grade 3 breast cancer was associated with inferior CSS. Further prospective data in this patient population are needed to confirm our findings and conclusions.

\section{Keywords}

High-grade; Omission; Oncology; Radiation; SEER

\section{Introduction}

For most women with invasive breast cancer, multiple randomized trials have established radiation therapy (RT) as the standard of care following breast conserving surgery (BCS). ${ }^{1-7}$ The Early Breast Cancer Trialists' Collaborative Group (EBCTCG) meta-analysis demonstrated that, in pathologically lymph node-negative women, not only was there a $15.4 \%$ (31.0\% without RT vs. $15.6 \%$ with RT) absolute reduction in any first recurrence at 10 years but also a $3.3 \%$ (20.5\% vs. $17.2 \%)$ absolute reduction in breast cancer-related death at 15 years. The absolute recurrence risk reduction of RT was less pronounced with increasing age, lower tumor grade, smaller tumors, and estrogen receptor (ER) positivity. ${ }^{6}$ Thus, many efforts have been made to identify patients who may be candidates for treatment de-escalation with the omission of RT.

NSABP B-21 was one of the earlier reported attempts to omit RT and showed no difference in overall survival (OS), but did show higher ipsilateral breast tumor recurrence (IBTR) rates in the group that did not receive RT. ${ }^{2}$ Additionally, a Canadian trial randomized 769 patients $\geq 50$ years with node-negative breast cancer to tamoxifen \pm RT after lumpectomy. They reported an 8-year local recurrence of $17.6 \%$ without RT and 3.5\% with RT. In an unplanned analysis, there was no significant difference in local control at 5 years in women $\geq 60$ years old with ER+ tumors $\leq 1 \mathrm{~cm}(1.2 \%$ vs. $0 \%){ }^{8}$

The effort to de-escalate led to CALGB 9343, which randomized 636 patients $\geq 70$ years of age with ER+, clinical stage I (cT1N0) breast cancer to tamoxifen \pm RT after lumpectomy. The long-term results with a median follow-up of 12 years demonstrated a 10-year local 
recurrence rate favoring RT (10\% vs. $2 \%$ ) without difference in OS, distant disease-free survival, or mastectomy-free survival. ${ }^{9}$ Similarly, PRIME II in the United Kingdom had a similar randomization, except the study included 1326 women $\geq 65$ years and tumors up to $3 \mathrm{~cm}$. With a median follow-up of 5 years, they reported an IBTR rate again favoring RT $(1.3 \%$ vs. $4.1 \%)$ without difference in regional recurrence or distant metastases. ${ }^{10}$

Taken together, these trials support the potential omission of RT in favorable-risk elderly women with breast cancer taking adjuvant endocrine therapy if the patient is willing to accept a relatively modest absolute increased risk of local recurrence. However, it is important to note that CALGB 9343 did not report histologic grade and PRIME II only included 36 (3\%) patients with grade 3 disease. Thus, the generalizability of these trials to elderly women with grade 3 disease remains unknown. Elderly women in general are more likely to present with less aggressive luminal type disease than younger patients. However, there is a significant proportion of these tumors that are luminal subtype B, which display a higher incidence of grade 3 disease and are associated with a poorer prognosis. ${ }^{11-13}$ Regardless, this treatment option has been incorporated into the National Comprehensive Cancer Network (NCCN) guidelines with a category 1 recommendation. ${ }^{14}$ A practice pattern analysis examining the Surveillance, Epidemiology, and End Results (SEER) database demonstrated a significant reduction in RT after the initial publication of CALGB $9343 .{ }^{15}$ This pattern was significant regardless of age, tumor size, and grade.

We hypothesized that high-grade disease may be unsuitable for treatment de-escalation and report the oncologic outcomes for select elderly women with favorable-risk early stage breast cancer treated with BCS with or without RT using the National Cancer Institute's SEER database.

\section{Materials and Methods}

\section{Database}

The SEER database contains comprehensive information concerning newly diagnosed cancers in individuals residing in 20 SEER-participating regions of the United States (US). The database is currently comprised of 18 registries, covering approximately $28 \%$ of the US population. Because the population-based data is provided in such a way that personal patient identification is not available, our study was exempt from institutional review board approval.

\section{Study Subjects}

In a retrospective study utilizing the SEER* Stat software version 8.3.4, we searched the SEER database of the US National Cancer Institute [18 Regs Research Data + Hurricane Katrina Impacted Louisiana Cases, Nov 2015 Sub (1973-2013 varying)] for patients diagnosed with breast cancer between 1997 and 2007. ${ }^{16}$ The initial cohort of 430,240 patients was then narrowed to 77,464 after only including patients between the ages of 70 and 79 years. We elected to exclude patients 80 years of age or older to decrease potential confounding secondary competing risks of death. ${ }^{17}$ Thus, the cohort was narrowed to 24,434 women with invasive ductal carcinoma histology (Site recode B International Classification 
of Diseases for Oncology, third revision 8500 Duct carcinoma) treated with BCS (Codes 21-24). The SEER definitions and codes are based on the American College of Surgeons Commission on Cancer FORDS surgery codes. BCS included lumpectomy or excisional biopsy (Code 22), re-excision of the biopsy site for gross or microscopic residual disease, partial mastectomy with nipple resection (Code 21), and segmental mastectomy (Code 24). This was then restricted to commensurate with the final CALBG inclusion criteria of women with tumors $\leq 20 \mathrm{~mm}$ T1N0M0 ER+ breast cancer treated with or without breast direct radiation, leaving a final cohort of 12,036 (Figure 1). Patients who received brachytherapy were excluded. Patients with contralateral breast intervention were excluded. Patients were included only if they were actively followed. Use of chemotherapy was not available in the database at the time when the data was acquired and thus, we were not able to use this as an exclusion factor.

\section{Endpoints}

Primary endpoints were 5-year and 10-year cause-specific survival (CSS). CSS was utilized to help mitigate the effects of selection bias between the non-RT and RT groups. CSS was measured as time from initial diagnosis until known death from breast cancer. Those who died of other causes or were alive at last follow-up were censored. Secondary endpoints were 5-year and 10-year OS.

\section{Covariates}

Covariates analyzed from the database include age at time of diagnosis, the year of diagnosis, grade, race, tumor size, and the receipt of RT. Human epidermal growth factor receptor 2 status was not available as it was not routinely collected in our cohort. Information on surgical margins, lymph vascular space invasion, and receipt of systemic therapy were not available in the SEER database at the time of analysis.

\section{Statistical Analysis}

Descriptive statistics were generated for each covariate, and tumor size was analyzed as both a continuous variable as well as a binary variable with a cutoff of $10 \mathrm{~mm}$. Numeric variables were summarized using mean, median, range, and standard deviation, and categorical variables were summarized using frequencies and percentages. Patient characteristics were compared across treatment groups (beam radiation vs. no radiation) using $\chi^{2}$ tests for categorical variables, and analysis of variance for numeric variables. CSS was estimated using the Kaplan-Meier method, and survival distributions were compared using log-rank tests. Univariate Cox proportional hazards models for CSS were fit with the above covariates, and a multivariable Cox model was fit for CSS as a function of radiation group, grade, T-stage, and age. Additionally, to reduce selection bias, we implemented a propensity score analysis using a matching algorithm for CSS within the grade 3 subgroup, where radiation patients were matched 1:1 to those with no radiation using a greedy matching approach. ${ }^{18,19}$ Variables included in the analysis were T-stage, age, and year of diagnosis, and variables were checked for balance. Model assumptions were checked and verified. The statistical analysis was performed using SAS 9.4 (SAS Institute Inc, Cary, NC), and the significance level was assessed at the 0.05 level. Significance level was not adjusted for multiple comparators in this exploratory analysis. 


\section{Results \\ Cohort Characteristics}

The final cohort included 12,036 women meeting criteria, with a median age of 74 years. Most (90\%) of the included patients were white. The median tumor size was $10 \mathrm{~mm}$. T1mic, T1a, T1b, and T1c comprised 1\%, 12\%, 39\%, and $48 \%$ of the cohort, respectively. Histologic grades 1, 2, and 3 comprised $36 \%, 50 \%$, and $14 \%$ of the cohort, respectively, and there were no differences in RT utilization between these subgroups (Table 1). RT was omitted in $22 \%$ of patients, including in $21 \%$ of patients with grade 3 disease. Patients in the RT cohort were slightly younger than the non-RT cohort (median 74 vs. 75 years; $P<.01$ ) and had fewer T1a tumors (11\% vs. $13 \% ; P=.02)$. Utilization of RT decreased following the initial publication of the CALGB 9343 trial in 2004, decreasing from $82 \%$ to $85 \%$ in 1998 to 2000 to $73 \%$ to $75 \%$ in 2005 to $2007(P<.01)$ (Figure 2). ${ }^{20}$

\section{Unadjusted Outcomes}

For the entire cohort, OS was $92.1 \%$ versus $83.5 \%$ at 5 years and $74.4 \%$ versus $62.3 \%$ at 10 years, favoring the radiation group $(P<.01)$ (Figure 3A). CSS was $98.0 \%$ versus $96.8 \%$ at 5 years and $95.1 \%$ versus $92.7 \%$ at 10 years, again favoring the group that received RT $(P$ $<.01$ ) (Figure 3B). On univariate analysis, receipt of radiation, T-stage, tumor size, grade, and age were significant predictors of CSS as detailed in Table 2. Race was not predictive of outcome. In grade 1 disease, there were no differences in CSS with or without RT at 5 years $(99 \%)$ and 10 years $(95 \%$ vs. $96 \%)(P=.1346)$. RT was associated with an improvement in CSS in grade 2 patients at 5 years (97\% vs. $98 \%)$ and at 10 years $(92 \%$ vs. $95 \%)(P=.004)$. In grade 3 disease, CSS improved from $93 \%$ to $96 \%$ at 5 years and from $87 \%$ to $92 \%$ at 10 years $(P=.02)$ with RT (Figure 4$)$. OS was also highly significant across all histologic subgroups, with the largest difference observed in the grade 3 population with an OS of $73.3 \%$ versus $59.9 \%$ at 10 years, favoring the radiation group.

\section{Adjusted Analysis}

A multivariable analysis was performed adjusting for receipt of radiation, grade, T-stage, and age. Each covariate remained a significant predictor of outcome as detailed in Table 3. Receipt of radiation was associated with significantly higher CSS (hazard ratio [HR], $0.68 ; 95 \%$ confidence interval [CI], 0.56-0.82; $P<.001]$. Propensity scored matching of age, T-stage, and year of diagnosis was then performed to compare the effect of RT on the high-grade cohort. In this high-grade subgroup, propensity score matching confirmed RT was associated with superior CSS compared with surgery alone: $91.8 \%$ versus $87.3 \%$ at 10 years (HR, 0.58; 95\% CI, 0.34-0.98; $P=.041$ ) (Table 4, Figure 5A). OS was also higher in the high-grade subgroup who received RT, $70.5 \%$ versus $59.9 \%$ at 10 years (HR, $0.63 ; 95 \%$ CI, 0.49-0.82; $P<.001$ ) (Figure 5B).

\section{Discussion}

Multiple randomized trials have established the role of RT in women with early stage breast cancer electing breast conservation therapy by demonstrating local control benefits. ${ }^{1-5,21,22}$ 
The EBCTCG meta-analysis additionally showed a decrease in the rate of breast cancerrelated death but also confirmed that many women are cured even without radiation.

As such, several efforts have been made to identify patients appropriate for treatment de-escalation. Patients with low-grade disease, ER positivity, smaller tumors, and women with increasing age are the ideal study group because data has shown less absolute benefit from RT in this cohort. For example, the absolute gain from radiation on the rate of any first recurrence at 10 years is $24.6 \%$ for women $<40$ years old and consistently declines to $8.9 \%$ after age 70.6

Two large randomized trials, CALGB 9343 and PRIME II, evaluated the omission of RT after BCS in elderly women with favorable-risk early stage breast cancer intending to take endocrine therapy. ${ }^{9,10}$ Both trials demonstrated a local control benefit of radiation, with no survival benefit. However, CALGB 9343 did not report histologic grade, and PRIME II only included 36 (3\%) patients with grade 3 disease.

The EBCTCG meta-analysis supports the concern that women $\geq 70$ years with high-grade disease are at a substantially increased risk of recurrence. In women $70+$ years with highgrade ER+ T1 tumors treated with tamoxifen, there was a $20 \%$ (27\% vs. $7 \%$ ) absolute risk reduction in any recurrence at 10 years when receiving radiation. ${ }^{6}$

Our study investigates whether the increased risk of local recurrence when omitting RT translates to a breast cancer-specific survival detriment in patients with high-grade disease. In our data, the use of adjuvant RT on unadjusted analysis was associated with higher CSS in the entire cohort. However, the benefit was not seen in the low-grade subgroup and was isolated to the intermediate and high-grade population. After applying propensity matching, a small but statistically significant CSS benefit remained in the group with high-grade disease (HR, 0.58; 95\% CI, 0.34-0.98; $P=.041$ ).

The finding that there was no CSS benefit in the low-grade population is in itself an important finding. In PRIME II, $42 \%$ of patients had low-grade disease, which can substantially influence the results of the entire cohort. Additionally, the omission of RT is predicated on adherence to adjuvant endocrine therapy, Poor adherence to endocrine therapy, which is estimated to be as high as $24 \%$ and worse in the low-income population, has been linked to worse outcomes. ${ }^{23,24}$ Thus, the benefit of RT may be more pronounced in the general population outside a clinical trial owing to known adherence issues with endocrine therapy secondary to adverse effects. ${ }^{25}$

Furthermore, clinical factors may not be the best predictors of when RT can safely be omitted. The TAILORx trial revolutionized the use of adjuvant chemotherapy via the use of a gene expression profile. ${ }^{26}$ There is a potential for gene expression profiling to more accurately determine the risk of recurrence and the benefits of adjuvant RT. ${ }^{27}$ For example, in the context of post-mastectomy RT, the Danish group and others have validated gene profiles predicting the benefit of RT. ${ }^{28,29}$ Histologic grade has been shown to be the strongest predictor of gene profile scores, far outweighing clinical stage and age. ${ }^{30}$ Therefore, high-grade disease may be more important than clinical factors in the favorablerisk, early stage population in selecting for RT. 
In the modern era, hypofractionated RT has made RT courses more convenient for the patient and less expensive for the payer without compromising outcomes. ${ }^{31,32}$ As a result, the relative value of RT is likely increased. Additionally, patients and referring providers often have preconceived concerns about the adverse effects of RT. One survey of 502 patients reported that $83 \%$ of patients stated that the acute severity of side effects were better than or as expected; similarly, $84 \%$ of patients reported that long-term side effects were better than or as expected. ${ }^{33}$ With modern RT techniques, most patients tolerate whole breast RT well with no to mild skin and subcutaneous late toxicity; however, a small, but variable percentage, ranging from $<10 \%$ to $25 \%$ of patients, can have persistent grade 2 to 3 late toxicities. ${ }^{31,32,34}$

Comorbidities are a significant competing cause of mortality in this population. We are not able to control for this variable in our study, which is why we included both CSS and OS and performed a matched analysis. The large difference in OS between the 2 groups likely demonstrates a substantial selection bias, which is likely owing to the presence of significant comorbidities. Clinically, it can be very difficult to know which patients will do well with their comorbidities versus those that will not. Patients who are thought to have a shorter life expectancy are undoubtably less likely to receive adjuvant radiation. As such, it is reasonable to assume that the group that did receive RT was without significant comorbidities.

Strengths of this study include the large sample size, which allowed for univariate, multivariate, and propensity matched analysis. The congruency of these models helps support the results that RT may be beneficial in select elderly patients with grade 3 disease. Limitations of the study include those inherent to database analyses, including selection bias. For example, we are not able to control for the reason a patient did not receive RT. Additionally, the SEER database in the above time frame does not include information regarding the receipt of adjuvant systemic therapy, adherence to systemic therapy, human epidermal growth factor receptor 2 status, lymph vascular space invasion, surgical margin status, or RT dose data.

\section{Conclusion}

Randomized studies support the omission of RT in elderly women with favorable-risk early stage breast cancer. However, grade 3 disease was not well represented in the CALGB or PRIME II trials, and it remains unclear whether omission of RT is supported for elderly patients with high-grade disease. Our study suggests there is an increased risk of breast cancer-specific death when omitting RT in select elderly women with high-grade disease, despite favorable-risk early stage breast cancer. Caution should be used when withholding RT, especially in women with high-grade disease and without competing comorbidities; thus, we encourage individualizing treatment with discussion between radiation oncologists and patients. Prospective data is needed to support our conclusions.

\section{Disclosure}

M. Torres reports employment with Malik Smith and research grants from the National Institutes of Health and Susan G. Komen. W.J. Curran reports research funding from AbbVie; honoraria from Bristol-Myers Squibb and 
AstraZeneca; and has acted as a paid consultant or in an advisory role for Varian Medical Systems and Vertex Pharmaceuticals for work performed outside of the current study. The remaining authors have stated that they have no conflicts of interest.

\section{References}

1. Fisher B, Anderson S, Bryant J, et al. Twenty-year follow-up of a randomized trial comparing total mastectomy, lumpectomy, and lumpectomy plus irradiation for the treatment of invasive breast cancer. N Engl J Med 2002; 347:1233-41. [PubMed: 12393820]

2. Fisher B, Bryant J, Dignam JJ, et al. , National Surgical Adjuvant Breast and Bowel Project. Tamoxifen, radiation therapy, or both for prevention of ipsilateral breast tumor recurrence after lumpectomy in women with invasive breast cancers of one centimeter or less. J Clin Oncol 2002; 20:4141-9. [PubMed: 12377957]

3. Veronesi U, Marubini E, Mariani L, et al. Radiotherapy after breast-conserving surgery in small breast carcinoma: long-term results of a randomized trial. Ann Oncol 2001; 12:997-1003. [PubMed: 11521809]

4. Holli K, Hietanen P, Saaristo R, Huhtala H, Hakama M, Joensuu H. Radiotherapy after segmental resection of breast cancer with favorable prognostic features: 12-year follow-up results of a randomized trial. J Clin Oncol 2009; 27:927-32. [PubMed: 19114687]

5. Forrest AP, Stewart HJ, Everington D, et al. Randomised controlled trial of conservation therapy for breast cancer: 6-year analysis of the Scottish trial. Scottish Cancer Trials Breast Group. Lancet 1996; 348:708-13. [PubMed: 8806289]

6. Early Breast Cancer Trialists' Collaborative Group (EBCTCG), Darby S, McGale P, Correa C, et al. Effect of radiotherapy after breast-conserving surgery on 10-year recurrence and 15-year breast cancer death: meta-analysis of individual patient data for 10,801 women in 17 randomised trials. Lancet 2011; 378:1707-16. [PubMed: 22019144]

7. Winzer KJ, Sauerbrei W, Braun M, et al. , German Breast Cancer Study Group (GBSG). Radiation therapy and tamoxifen after breast-conserving surgery: updated results of a $2 \times 2$ randomised clinical trial in patients with low risk of recurrence. Eur J Cancer 2010; 46:95-101. [PubMed: 19879131]

8. Fyles AW, McCready DR, Manchul LA, et al. Tamoxifen with or without breast irradiation in women 50 years of age or older with early breast cancer. N Engl J Med 2004; 351:963-70. [PubMed: 15342804]

9. Hughes KS, Schnaper LA, Bellon JR, et al. Lumpectomy plus tamoxifen with or without irradiation in women age 70 years or older with early breast cancer: long-term follow-up of CALGB 9343. J Clin Oncol 2013; 31:2382-7. [PubMed: 23690420]

10. Kunkler IH, Williams LJ, Jack WJL, Cameron DA, Dixon JM, PRIME II investigators. Breastconserving surgery with or without irradiation in women aged 65 years or older with early breast cancer (PRIME II): a randomised controlled trial. Lancet Oncol 2015; 16:266-73. [PubMed: 25637340]

11. de Kruijf EM, Bastiaannet E, Rubertá F, et al. Comparison of frequencies and prognostic effect of molecular subtypes between young and elderly breast cancer patients. Mol Oncol 2014; 8:101425. [PubMed: 24767310]

12. Durbecq V, Ameye L, Veys I, et al. A significant proportion of elderly patients develop hormone-dependant "luminal-B" tumours associated with aggressive characteristics. Crit Rev Oncol Hematol 2008; 67:80-92. [PubMed: 18313937]

13. Engels CC, Kiderlen M, Bastiaannet E, et al. The clinical prognostic value of molecular intrinsic tumor subtypes in older breast cancer patients: a FOCUS study analysis. Mol Oncol 2016; 10:594600. [PubMed: 26706834]

14. National Comprehensive Cancer Network. Breast Cancer (Version 2.2017) 10 4, 2017, Available at: www.nccn.org/professionals/physician_gls/pdf/breast.pdf. Accessed: October 4, 2017.

15. Palta M, Palta P, Bhavsar NA, Horton JK, Blitzblau RC. The use of adjuvant radiotherapy in elderly patients with early-stage breast cancer: changes in practice patterns after publication of Cancer and Leukemia Group B 9343. Cancer 2015; 121:188-93. [PubMed: 25488523] 
16. National Institutes of Health; National Cancer Institute. Surveillance, Epidemiology, and End Results (SEER) Program. SEER*Stat Database: Incidence -SEER 18 Regs Research Data + Hurricane Katrina Impacted Louisiana Cases, Nov 2013 Sub (1973-2011 varying) -Linked To County Attributes -Total U.S., 1969-2012 Counties, National Cancer Institute, DCCPS, Surveillance Research Program, Surveillance Systems Branch, released April 2014 (updated 5/7/2014), based on the November 2013 submission, Available at: www.seer.cancer.gov. Accessed: February 21, 2015.

17. Smith BD, Gross CP, Smith GL, Galusha DH, Bekelman JE, Haffty BG. Effectiveness of radiation therapy for older women with early breast cancer. J Natl Cancer Inst 2006; 98:681-90. [PubMed: 16705122]

18. Rosenbaum P, Rubin D. The central role of the propensity score in observational studies for causal effects. Biometrika 1983; 70:41-55.

19. Rosenbaum PR. Observational studies. Springer series in statistics New York: Springer-Verlag; 1995.

20. Hughes KS, Schnaper LA, Berry D, et al. , Cancer and Leukemia Group B; Radiation Therapy Oncology Group; Eastern Cooperative Oncology Group. Lumpectomy plus tamoxifen with or without irradiation in women 70 years of age or older with early breast cancer. N Engl J Med 2004; 351:971-7. [PubMed: 15342805]

21. Liljegren G, Holmberg L, Bergh J, et al. 10-Year results after sector resection with or without postoperative radiotherapy for stage I breast cancer: a randomized trial. J Clin Oncol 1999; 17:2326-33. [PubMed: 10561294]

22. Clark RM, Whelan T, Levine M, et al. Randomized clinical trial of breast irradiation following lumpectomy and axillary dissection for node-negative breast cancer: an update. Ontario Clinical Oncology Group. J Natl Cancer Inst 1996; 88:1659-64. [PubMed: 8931610]

23. Makubate B, Donnan PT, Dewar JA, Thompson AM, McCowan C. Cohort study of adherence to adjuvant endocrine therapy, breast cancer recurrence and mortality. Br J Cancer 2013; 108:151524. [PubMed: 23519057]

24. Hershman DL, Tsui J, Wright JD, Coromilas EJ, Tsai WY, Neugut AI. Household net worth, racial disparities, and hormonal therapy adherence among women with early-stage breast cancer. J Clin Oncol 2015; 33:1053-9. [PubMed: 25691670]

25. Kidwell KM, Harte SE, Hayes DF, et al. Patient-reported symptoms and discontinuation of adjuvant aromatase inhibitor therapy. Cancer 2014; 120:2403-11. [PubMed: 24802413]

26. Sparano JA, Gray RJ, Makower DF, et al. Adjuvant chemotherapy guided by a 21-gene expression assay in breast cancer. N Engl J Med 2018; 379:111-21. [PubMed: 29860917]

27. Nuyten DS, Kreike B, Hart AMA, et al. Predicting a local recurrence after breast-conserving therapy by gene expression profiling. Breast Cancer Res 2006; 8:R62. [PubMed: 17069664]

28. Tramm T, Mohammed H, Myhre S, et al. Development and validation of a gene profile predicting benefit of postmastectomy radiotherapy in patients with high-risk breast cancer: a study of gene expression in the DBCG82bc cohort. Clin Cancer Res 2014; 20:5272-80. [PubMed: 25149560]

29. Cheng SH, Horng CF, Huang TT, et al. An eighteen-gene classifier predicts locoregional recurrence in post-mastectomy breast cancer patients. EBioMedicine 2016; 5:74-81. [PubMed: 27077114]

30. Turner BM, Skinner KA, Tang P, et al. Use of modified Magee equations and histologic criteria to predict the Oncotype DX recurrence score. Mod Pathol 2015; 28:921-31. [PubMed: 25932962]

31. Whelan TJ, Pignol JP, Levine MN, et al. Long-term results of hypofractionated radiation therapy for breast cancer. N Engl J Med 2010; 362:513-20. [PubMed: 20147717]

32. Haviland JS, Owen JR, Dewar JA, et al. , START Trialists' Group. The UK Standardisation of Breast Radiotherapy (START) trials of radiotherapy hypofractionation for treatment of early breast cancer: 10-year follow-up results of two randomised controlled trials. Lancet Oncol 2013; 14:1086-94. [PubMed: 24055415]

33. Shaverdian N, Wang X, Hegde JV, et al. The patient's perspective on breast radiotherapy: initial fears and expectations versus reality. Cancer 2018; 124: 1673-81. [PubMed: 29479673]

34. Goldsmith C, Haviland J, Tsang Y, et al. , FAST Trialists' Group. Large breast size as a risk factor for late adverse effects of breast radiotherapy: is residual dose inhomogeneity, despite 
3D treatment planning and delivery, the main explanation? Radiother Oncol 2011; 100:236-40.

[PubMed: 21296441] 


\section{Clinical Practice Points}

- $\quad$ Randomized studies support the omission of RT in elderly women with favorable-risk early stage breast cancer. However, grade 3 disease was not well represented in the CALGB or PRIME-II trials, and it remains unclear whether omission of RT is supported for elderly patients with high-grade disease.

- We performed a SEER analysis of 12,036 women aged 70 to 79 years with T1N0, ER+ IDC treated with BCS. In this study, women with grade 3 disease had an improvement in breast cancer-specific survival from $93 \%$ to $96 \%$ at 5 years and from $87 \%$ to $92 \%$ at 10 years $(P=.02)$ with RT. OS was also highly significant across all histologic subgroups, with the largest difference observed in the grade 3 population with an OS of $73.3 \%$ versus $59.9 \%$ at 10 years favoring the radiation group. Propensity score matching was performed in the grade 3 cohort, and again, RT was associated with superior breast cancer-specific survival compared with surgery alone $(91.8 \%$ vs. $87.3 \%$ at 10 years; HR, $0.58 ; P=.041]$.

- Our study suggests there is an increased risk of breast cancer-specific death when omitting RT in select elderly women with high-grade disease, despite favorable-risk early stage breast cancer.

- We suggest that caution should be used when withholding RT, especially in women with high-grade disease and without competing comorbidities. Treatment should be individualized, and women in this population should be encouraged to discuss the risks and benefits of treatment with a radiation oncologist. Prospective data will be needed for this high-grade population to support these findings. 


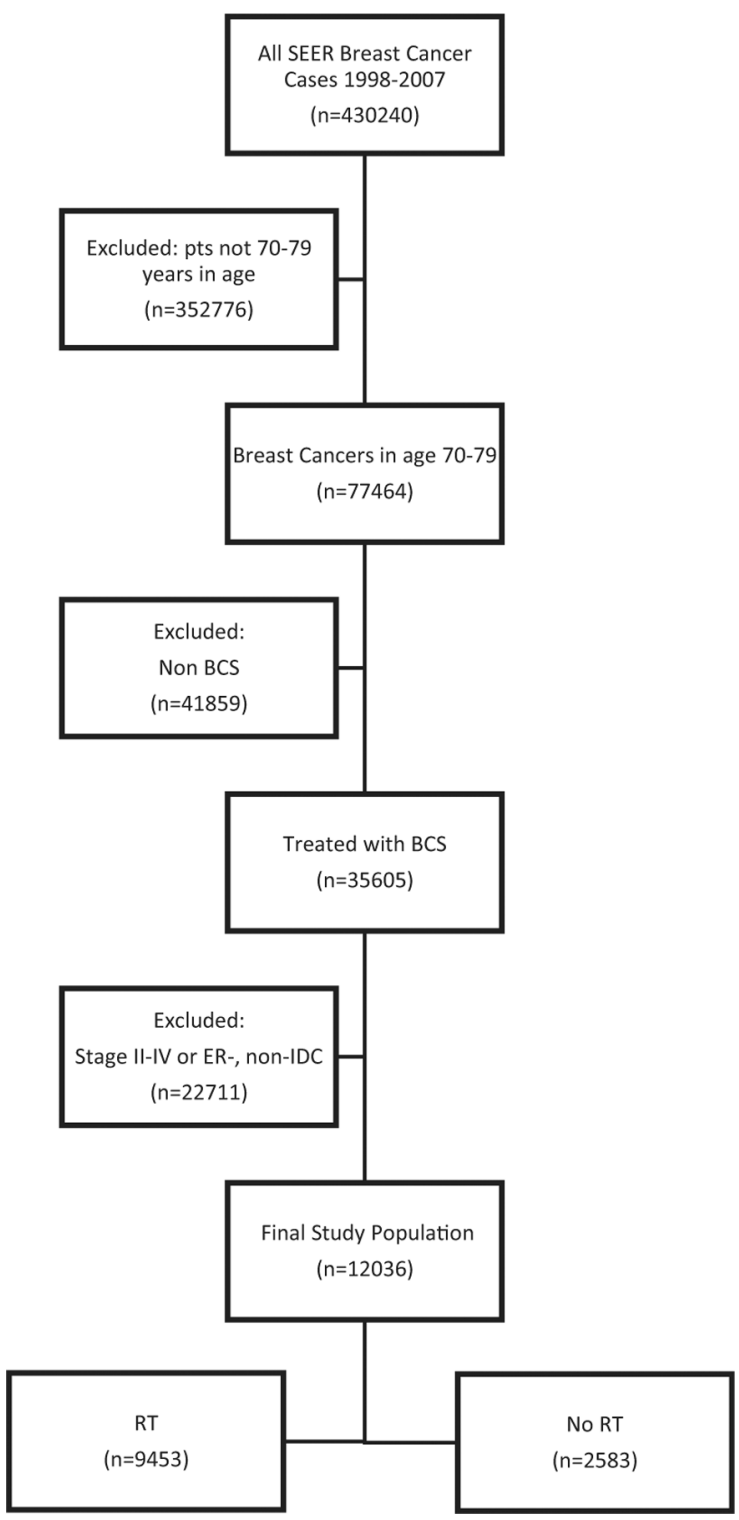

Figure 1.

Selection/Exclusion Diagram

Abbreviations: $\mathrm{BCS}=$ breast conserving surgery; $\mathrm{ER}^{-}=$estrogen receptor-negative; IDC $=$ invasive ductal carcinoma; $\mathrm{pts}=$ patients RT $=$ radiation therapy SEER $=$ Surveillance, Epidemiology, and End Results. 
RT UTILIZATION, 1998 - 2007

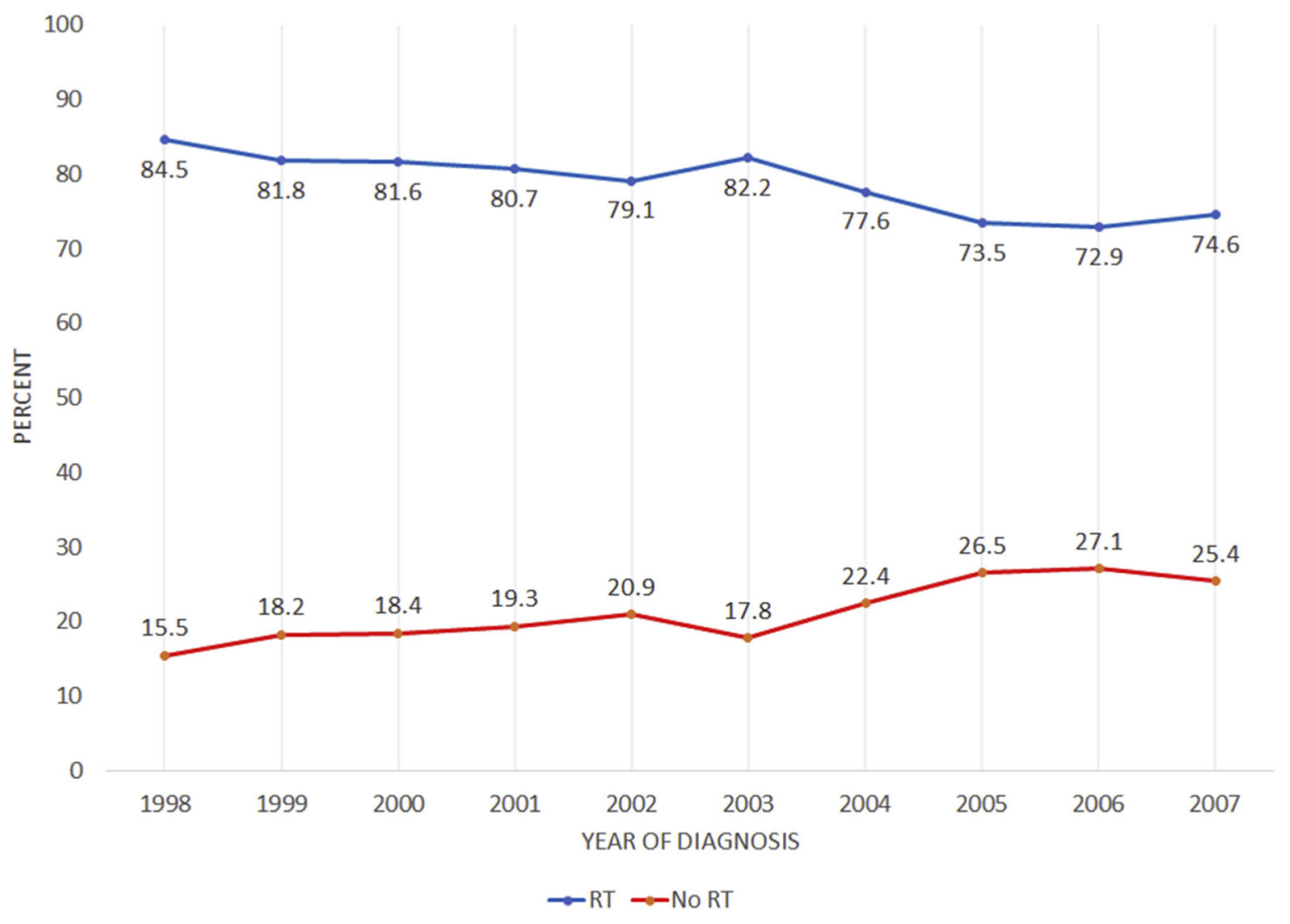

Figure 2.

Trend of RT Utilization Through the Study Period, 1998-2007 

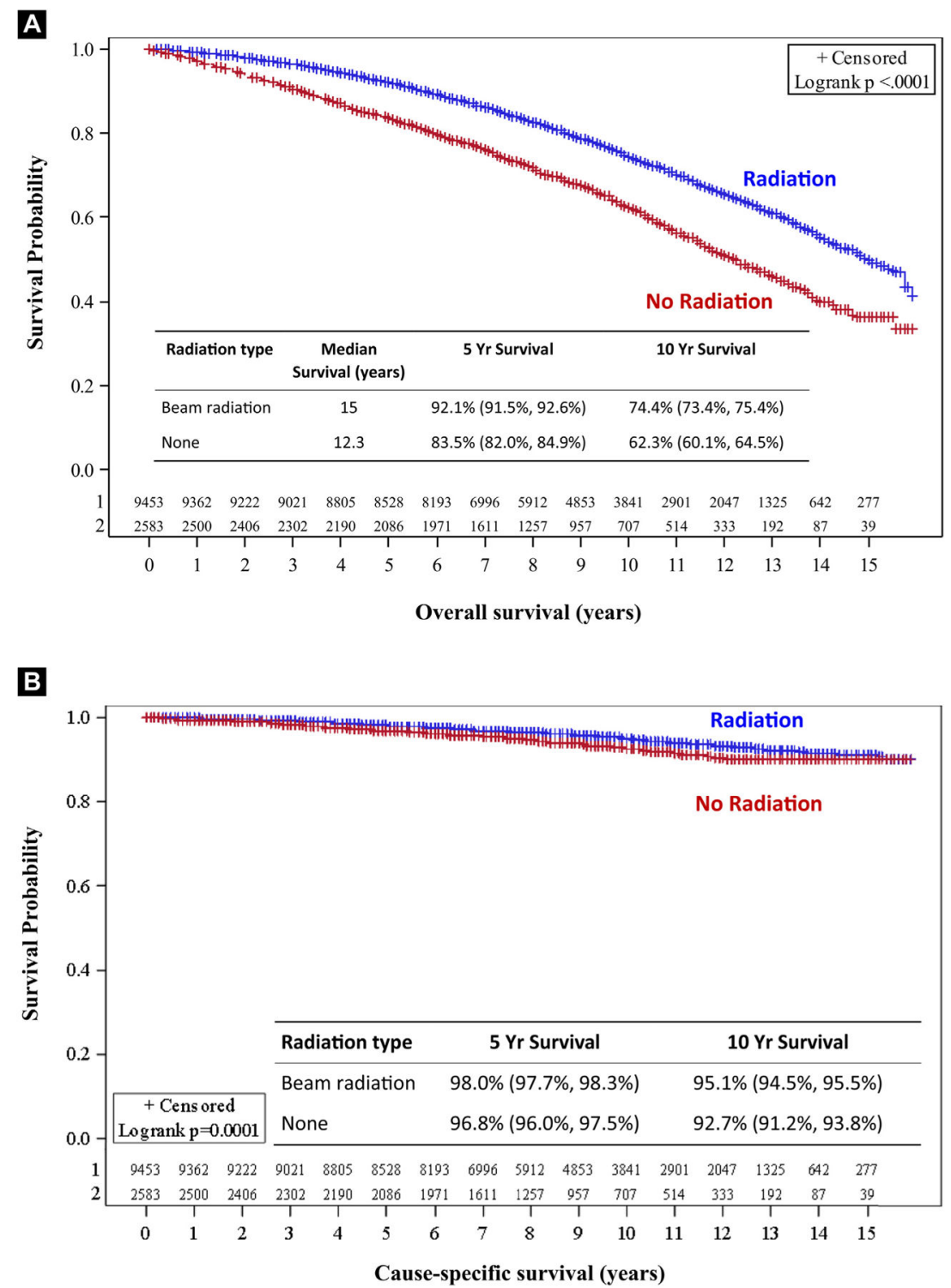

Figure 3.

Overall Survival (A) and Cause-specific Survival (B) Based on Receipt of Adjuvant Radiation Following Breast Conserving Surgery 

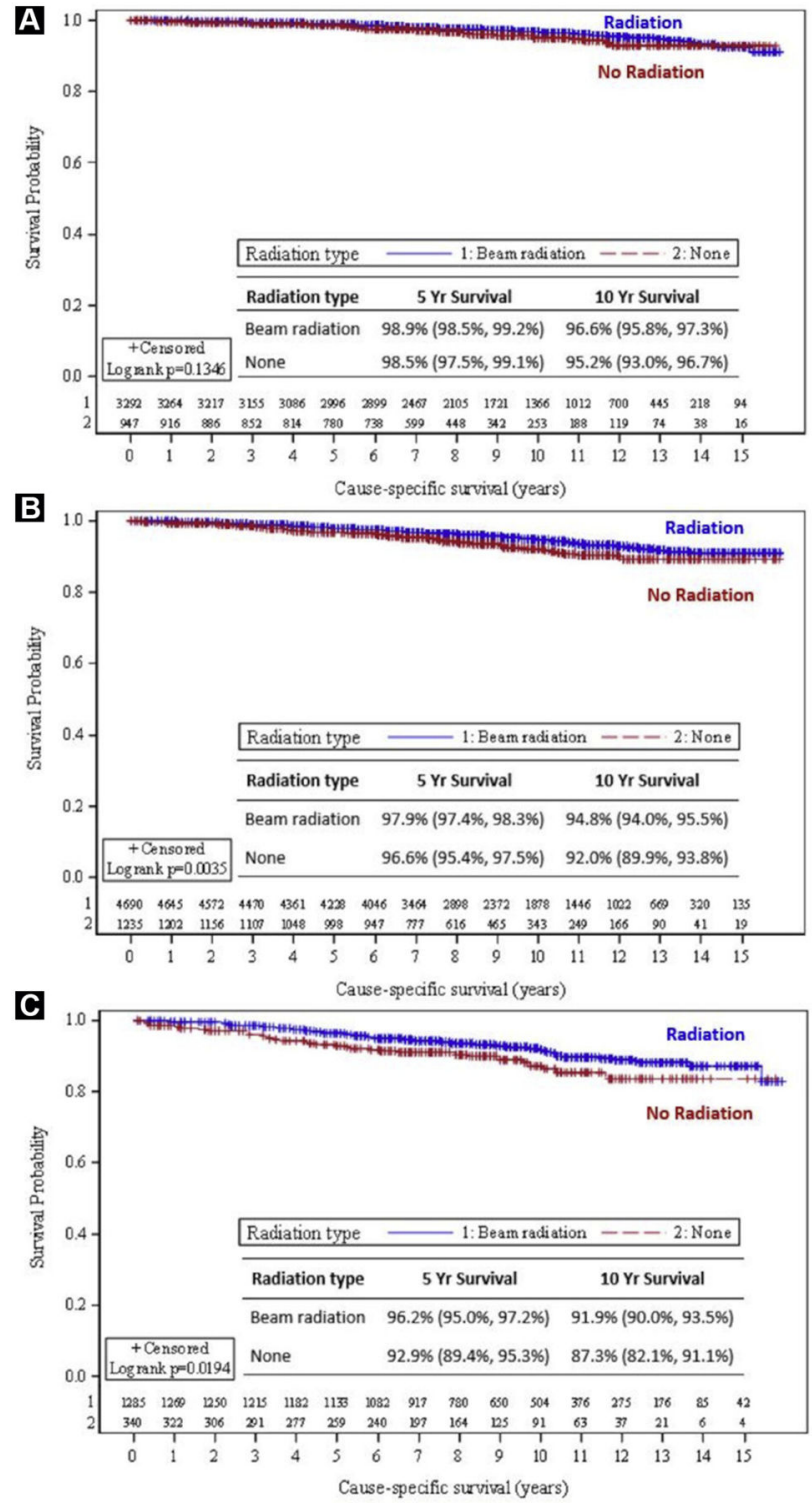

Figure 4.

Cause-specific Survival Stratified by Histology. A, Low-Grade Histology; B, IntermediateGrade Histology; C, High-Grade Histology 
A

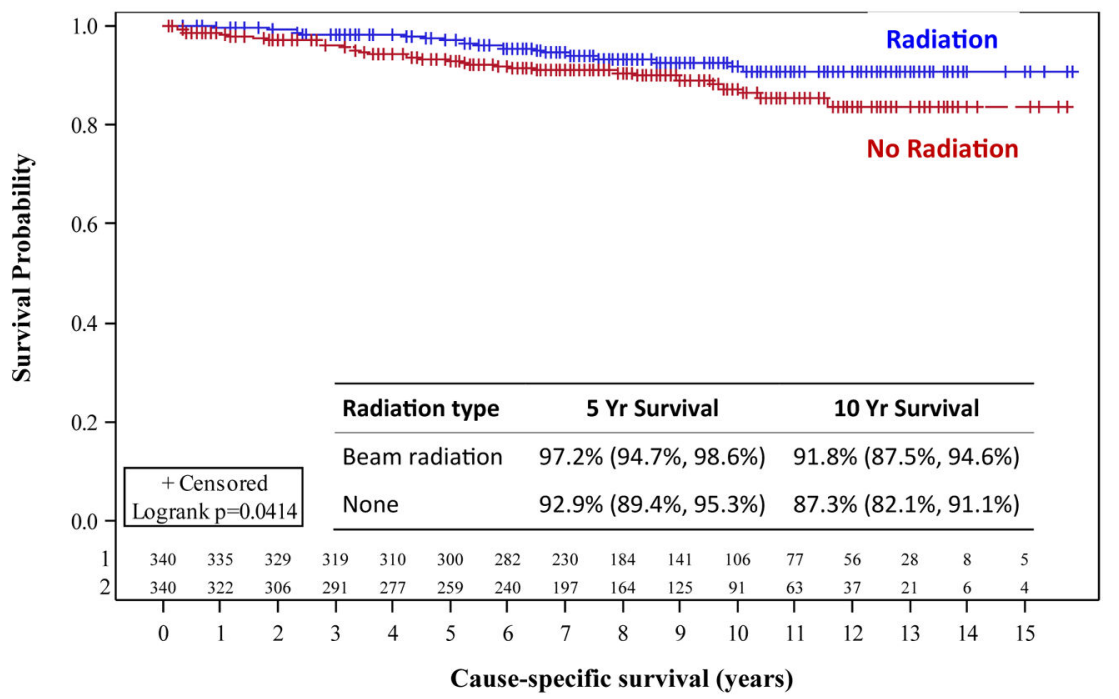

B

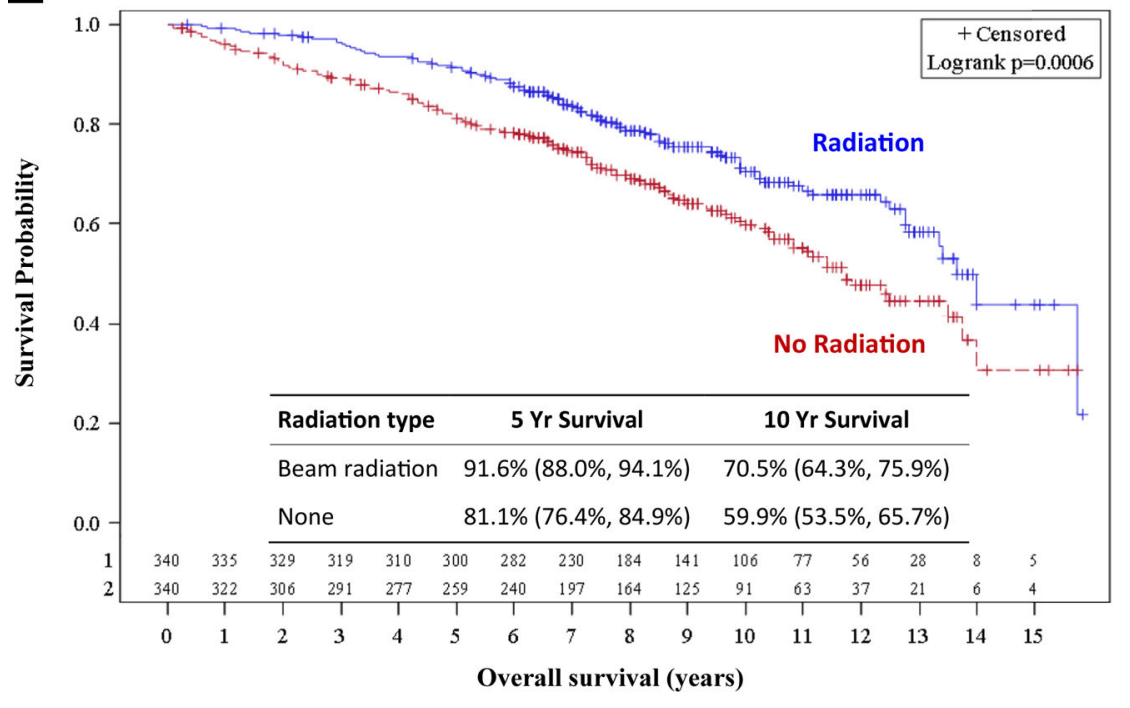

Figure 5.

High Grade - Propensity Matched Groups. A, Cause-specific Survival; B, Overall Survival 
Table 1

Treatment Group Comparison

\begin{tabular}{|c|c|c|c|}
\hline \multirow[b]{2}{*}{ Covariate } & \multicolumn{2}{|c|}{ Radiation, $\mathbf{n}(\%)$} & \multirow[b]{2}{*}{$P$ Valu } \\
\hline & None $\mathbf{N}=\mathbf{2 5 8 3}$ & Beam Radiation N = 9453 & \\
\hline Age, y & & & $<.001$ \\
\hline Median & 75 & 74 & \\
\hline Race & & & .528 \\
\hline White & $2321(90.21)$ & $8480(89.78)$ & \\
\hline Other & $252(9.79)$ & $965(10.22)$ & \\
\hline Year of diagnosis & & & $<.001$ \\
\hline 1998 & $120(4.65)$ & $656(6.94)$ & \\
\hline 1999 & $143(5.54)$ & $643(6.8)$ & \\
\hline 2000 & $251(9.72)$ & $1114(11.78)$ & \\
\hline 2001 & $257(9.95)$ & $1073(11.35)$ & \\
\hline 2002 & $260(10.07)$ & $1032(10.92)$ & \\
\hline 2003 & $223(8.63)$ & $1028(10.87)$ & \\
\hline 2004 & $283(10.96)$ & $980(10.37)$ & \\
\hline 2005 & $344(13.32)$ & $956(10.11)$ & \\
\hline 2006 & $359(13.9)$ & $966(10.22)$ & \\
\hline 2007 & $343(13.28)$ & $1005(10.63)$ & \\
\hline $\mathrm{T}$ stage & & & .015 \\
\hline T1a & $341(13.2)$ & $1048(11.09)$ & \\
\hline $\mathrm{T} 1 \mathrm{~b}$ & $1002(38.79)$ & $3738(39.54)$ & \\
\hline $\mathrm{T} 1 \mathrm{c}$ & $1205(46.65)$ & $4564(48.28)$ & \\
\hline T1mic & $35(1.36)$ & $103(1.09)$ & \\
\hline Tumor size, mm & & & .142 \\
\hline Mean & 10.71 & 10.88 & \\
\hline Median & 10 & 10 & \\
\hline$\leq 10$ & $1378(53.35)$ & $4889(51.72)$ & .089 \\
\hline$>10$ & $1205(46.65)$ & $4564(48.28)$ & \\
\hline Grade & & & .171 \\
\hline Low & $947(37.55)$ & $3292(35.52)$ & \\
\hline Intermediate & $1235(48.97)$ & $4690(50.61)$ & \\
\hline High & $340(13.48)$ & $1285(13.87)$ & \\
\hline
\end{tabular}

Bold values are significant. 


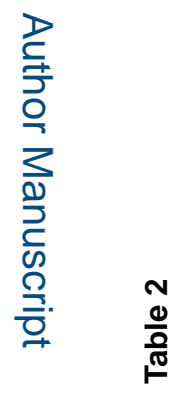

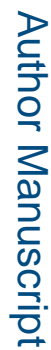

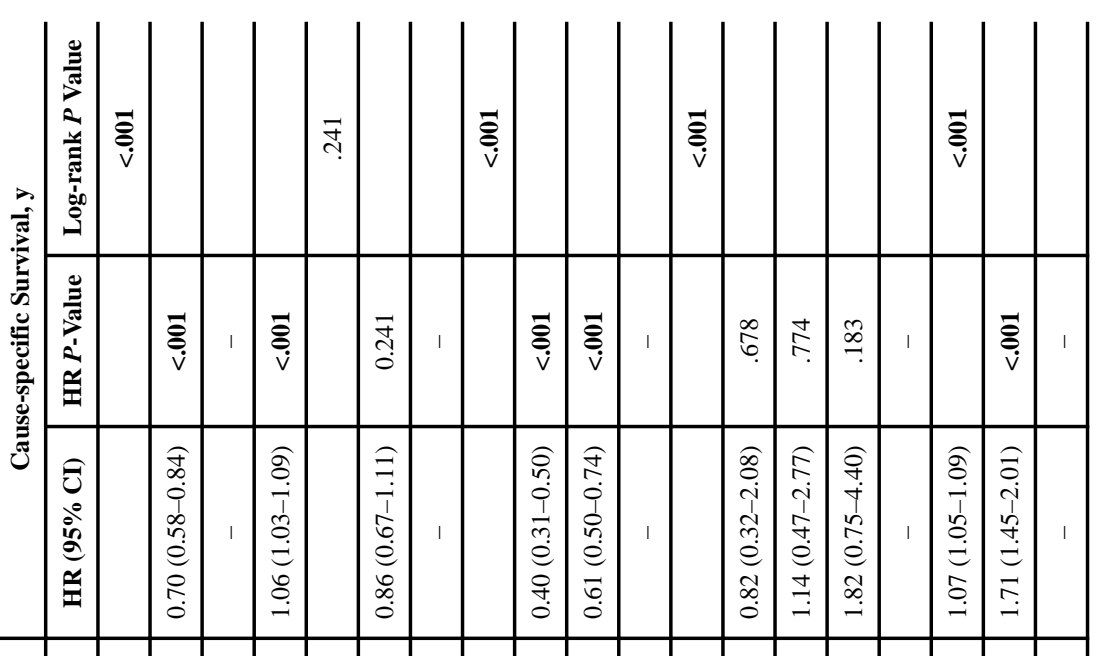

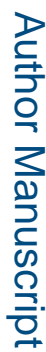

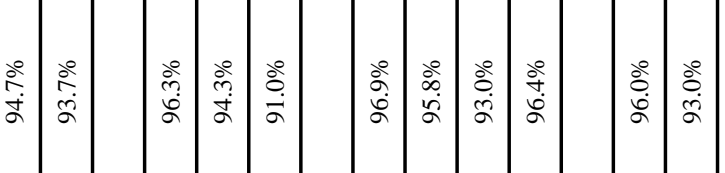

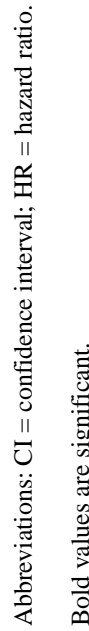

Clin Breast Cancer. Author manuscript; available in PMC 2021 October 07. 
Table 3

Multivariate Analysis: Cause-specific Survival

\begin{tabular}{l|c|c|c} 
& \multicolumn{3}{|c}{ Cause-specific Survival, y } \\
\hline Covariate & HR $(\mathbf{9 5 \%}$ CI $)$ & HR $\boldsymbol{P}$ Value & Wald $\boldsymbol{P}$ Value \\
\hline Radiation type & & & \\
\hline Beam radiation & $0.68(0.56-0.82)$ & $<.001$ & $<.001$ \\
\hline None & - & - & \\
\hline Age & $1.05(1.02-1.08)$ & $<.001$ & $<.001$ \\
\hline Grade & & & \\
\hline Low & $0.45(0.35-0.57)$ & $<.001$ & $<.001$ \\
\hline Intermediate & $0.63(0.52-0.78)$ & $<.001$ & \\
\hline High & - & - & \\
\hline T stage & & & \\
\hline T1a & $0.98(0.35-2.74)$ & .968 & $<.001$ \\
\hline T1b & $1.34(0.50-3.62)$ & .559 & \\
\hline T1c & $2.00(0.74-5.35)$ & .169 & \\
\hline T1mic & - & - & \\
\hline
\end{tabular}

Abbreviations: $\mathrm{CI}=$ confidence interval; $\mathrm{HR}=$ hazard ratio.

Bold values are significant. 
Table 4

High Grade: Propensity Matched Groups: Cause-specific Survival

\begin{tabular}{l|c|c|c|c} 
& & \multicolumn{3}{|c}{ Cause-specific Survival, $\mathbf{~}$} \\
\hline Covariate & N & HR $(\mathbf{9 5 \%}$ CI $)$ & HR P-value & Log-rank $\boldsymbol{P}$ Value \\
\hline Radiation type & & & & \\
\hline Beam radiation & 340 & $0.58(0.34-0.98)$ & $\mathbf{. 0 4 3}$ & $\mathbf{. 0 4 1}$ \\
\hline None & 340 & - & - & \\
\hline
\end{tabular}

Abbreviations: $\mathrm{CI}=$ confidence interval; $\mathrm{HR}=$ hazard ratio

Bold values are significant. 